\title{
A mathematical model to predict early quality attributes in hake during storage at low temperature
}

\author{
C. Vilas ${ }^{a}$, A. A. Alonso ${ }^{a *}$, J.R. Herrera ${ }^{b}$, M. Bernárdez ${ }^{b}$, M.R.

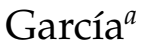 \\ ${ }^{a}$ Bioprocess Engineering Group. IIM-CSIC. c/ Eduardo Cabello,6. 36208 Vigo, Spain \\ ${ }^{b}$ Microbiology and Technology of Marine Products, IIM-CSIC, c/ Eduardo Cabello 6, 36208, \\ Vigo, Spain
}

\begin{abstract}
In this work we develop a model describing the evolution of the adenosine triphosphate (ATP) degradation products, typically used as early indicators of fish quality loss, during storage and transport conditions. The model is constructed following a modular approach that includes essentially three mechanisms: (1) enzymatic transformation of inosine 5'monophosphate (IMP), inosine (Ino) and hypoxanthine (Hx) with some reactions catalyzed by bacteria; (2) bacterial growth and (3) nucleotide diffusion through the food matrix. This approach allows us to combine the different underlying mechanisms to account for other fish species and conditions.

We compare alternative mechanisms explaining the catalytic effect of Pseudomonas and Shewanella populations on the reaction linking IMP, Ino and Hx. The selection is carried out in terms of the Akaike Information Criteria.

The predictive capabilities of the selected model are demonstrated with experiments.
\end{abstract}

Keywords: Fish freshness; Early indicators; Parameter estimation; Nucleotide degradation; Dynamic model; Bacterial catalysis

\section{Introduction}

Freshness is one of the most important attributes to define the market value of fish (García et al. 2017). Loss of fish acceptability is generally due to bacterial

${ }^{*}$ Corresponding author: antonio@iim.csic.es Postprint submitted to Journal of Food Engineering 
spoilage (Hong et al. 2017) which occurs several days after fish death at typical storage conditions. However, loss of fish freshness starts early after fish death and before bacterial spoilage can be clearly noticed. Abundance of adenosine triphosphate (ATP) degradation compounds has been widely used as a reliable indicator to assess fish freshness at early storage stages (Saito et al., 1959: Hara and Uda. 1984 Hong et al. 2017). Among the degradation compounds, inosine 5'-monophosphate (IMP) provides a sweet and meaty flavor that contributes to enhance fish quality whereas its conversion in hypoxanthine $(\mathrm{Hx})$ is responsible of unpleasant bitterness (Surette et al., 1988; Haard, 2002, Kawai et al., 2002, Kuda et al. 2008; Li et al. 2015). The $K_{I}$-index (Karube et al., 1984), that relates concentration of IMP, inosine (Ino) and $\mathrm{Hx}$, has been proposed as a reliable freshness indicator. The $K_{I}$-index is defined as:

$$
K_{I}(\%)=\frac{[\mathrm{Ino}]+[\mathrm{Hx}]}{[\mathrm{IMP}]+[\mathrm{Ino}]+[\mathrm{Hx}]}
$$

In this way, studying the degradation pathways of IMP into Ino and Hx will allow us to derive mathematical models that can be used to monitor and predict quality losses of fish at early storage times.

IMP degradation under sterile conditions has been studied by several authors (Davídek et al. 1972, Hara and Uda, 1984, Howgate, 2006). Recently, Vilas et al. (2017a) proposed a biochemical pathway that explained nucleotide degradation kinetics in European hake (Merluccius merluccius) under sterile conditions. In that work, the authors started from the complete enzymatic degradation scheme presented in Figure 2, determined the relevant reactions by fitting the model to experimental data, and reduced the original scheme to the one presented in Figure 3 by neglecting non relevant reactions.

Under regular storage conditions, and in addition to physical barriers that may limit enzyme accessibility or nucleotide dispersion through the fish muscle, the dynamics of IMP degradation is, at a high extent, influenced by the presence of bacteria (Surette et al., 1988: Howgate, 2006: Zotos, 2010: Li et al. 2017). To illustrate this point, Figure 1(a) presents the evolution of IMP and Hx for two experiments performed under sterile and non-sterile conditions. Both 
experiments were carried out under similar storage temperatures which lied in the order of $6{ }^{\circ} \mathrm{C}$ (Figure $1 \mathrm{~b}$ )). As shown in the figure the rates of IMP degrada-

(a)

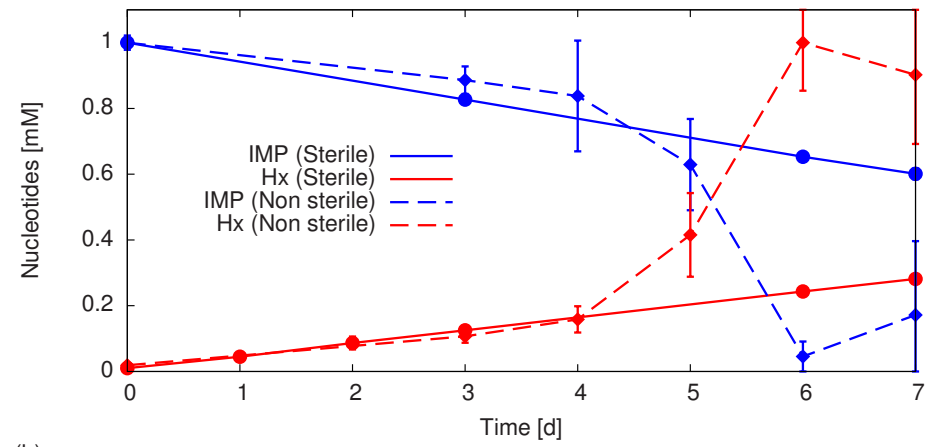

(b)

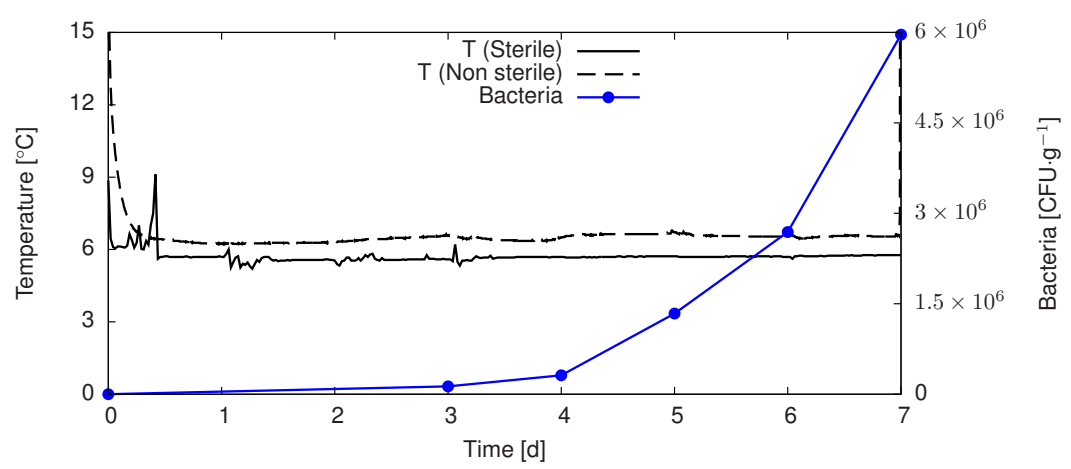

Figure 1: (a) Evolution of IMP and Hx in sterile conditions (continuous lines) and in the presence of bacteria (dashed lines). (b) Bacteria concentration (Pseudomonas plus Shewanella) in the non-sterile experiment and temperature in the experiments carried out under sterile (continuous line) and non-sterile conditions (dashed line)

tion and $\mathrm{Hx}$ formation increase considerably as bacteria concentration becomes significant (from approximately day 4 ) in the non sterilized experiment. This suggests a bacterial catalytic action.

In this regard, alkaline phosphatase and 5'-nucleotidase, the major enzymes involved in IMP degradation, are widespread in bacteria (Zimmermann, 1992); including Shewanella (Ishida et al. 1998: Tsuruta et al. 2010: Kuribayashi et al., 2017) and Pseudomonas (Lazdunski A, 1990, Beveridge and Kadurugamuwa, 1996). Ino can turn to Hx by purine nucleoside phosphorylase and inosine nucleosidase, which are also widely distributed in bacteria, including spoilage 
organisms of fresh fish such as Shewanella and Pseudomonas, as found in protein databases (e.g. UniProt, STRING). Previously, Surette et al. (1988) suggested that Pseudomonas was responsible for the production of intracellular inosine nucleosidase in fresh fish. We assume that Pseudomonas and Shewanella are the predominant bacterial species involved in the spoilage of fresh fish (Gram and Dalgaard, 2002, Gram and Huss, 1996) and make use of the model developed by García et al. (2015) to describe their growth as a function of temperature.

Competition among bacteria may constitute an important issue to be considered. Modeling such competition has been addressed in a number of works (Giuffrida et al. 2007, 2009a b), typically using Lotka-Volterra models. We assume that, for the bacteria considered in the present study, competition is not relevant for the following reasons: (i) the growth of Pseudomonas in fish stored at low temperature is unaffected by the presence of Shewanella (Gram and Melchiorsen, 1996); (ii) the growth of Shewanella is only affected at high cell densities of Pseudomonas (Gram and Melchiorsen, 1996), that are not reached in the present study; (iii) most Pseudomonas isolated from fish (over $80 \%$ ) did not inhibit Shewanella under ice-stored conditions (Gram, 1993). This assumption has been previously validated in García et al. (2015).

Environmental variables such as temperature, $\mathrm{pH}$, or water activity may affect bacterial growth (Giuffrida et al., 2009b). Water activity remains virtually constant, whereas $\mathrm{pH}$ in the case of hake only experiences slight changes around neutrality, where the activity of Pseudomonas and Shewanella is expected to be hardly affected. Temperature is, to a large extent, the most important variable that may also change during the storage of fresh fish. In consequence, we focus on the effect of temperature on bacterial growth and enzymatic transformations.

In this work we propose a model that predicts the evolution of ATP degradation products (IMP, Ino and $\mathrm{Hx}$ ), in fish muscle during storage at low temperatures. The model incorporates the effect of bacterial concentrations (Pseudomonas and Shewanella) on the reaction rates associated to the biochemical pathway proposed in Vilas et al. (2017a). As suggested in Howgate (2006), a first order leakage term is included to consider nucleotide diffusion in fish 
muscle. This approach allows us to combine, in a modular way, the different underlying mechanisms to account for different fish matrices and storage conditions.

Unknown model parameters are estimated by fitting the model to the experimental data. In order to test their predictive capabilities, the model is validated on a set of experiments that reproduce typical storage conditions.

\section{Experimental methods}

\subsection{Fish handling and storage conditions}

Fresh gutted medium-sized hake (Merluccius merluccius) (400-500 g) caught in Galician waters either by bottom-set or long-line were purchased from the retail market in Vigo (Spain) during the first $24 \mathrm{~h}$ after slaughtering. Hake was transferred to the laboratory within $30 \mathrm{~min}$ in expanded polystyrene boxes with ice.

For storage trials, ice was completely removed and boxes containing hake were sealed and stored under refrigeration conditions during 7-12 d either on an incubator (Model EC-570, Radiber S.A.) for experiments at $T \geq 5^{\circ} \mathrm{C}$, or on a KIDE universal cold room for experiments at $T<5^{\circ} \mathrm{C}$. Four experiments were performed using gutted hake captured by bottom-set nets. In three of these experiments, the refrigeration temperature was fixed to $1,2.5$ and $6.5^{\circ} \mathrm{C}$. In the last experiment, temperature during the first seven days was fixed to 0.5 ${ }^{\circ} \mathrm{C}$, then it was increased to $11^{\circ} \mathrm{C}$. A thermocouple was inserted within the abdominal cavity of one fish to record the temperature every 5 min throughout the storage period. Fishes were taken out of refrigerated storage on a daily basis. Each fish was used to prepare one sample. In the experiments carried out at $1,2.5$ and $6.5^{\circ} \mathrm{C}$ three samples were prepared at the beginning of each experiment $(t=0)$ whereas two fishes were used at the remaining sampling times. In the experiment carried out at 0.5 and $11^{\circ} \mathrm{C}$ four fishes were used per sampling time. 


\subsection{Nucleotide catabolites and bacteria analysis}

The analysis is based on the method described in Özogul et al. (2000). Briefly, $5.0 \mathrm{~g}$ of fish muscle were homogenized in $25 \mathrm{~mL}$ of cold $0.6 \mathrm{M}$ perchloric acid by using a tissue blender (Ultra-Turrax, IKA Werke GmbH \& Co., Staufen, Germany) at maximum speed $(2 \times 30 \mathrm{~s})$. Homogenization was carried out in a beaker placed in an ice-water bath to prevent any rise of temperature.

Homogenates were stored at $-20^{\circ} \mathrm{C}$ for $10 \mathrm{~min}$ and then centrifuged at 1000 $\mathrm{g}$ for $10 \mathrm{~min}$ at $4{ }^{\circ} \mathrm{C}$. Supernatants were collected and $1 \mathrm{M}$ potassium hydroxide was immediately added up to reaching a pH between 6.5-6.8. Once neutralized, homogenates were kept for $30 \mathrm{~min}$ in an ice-water bath for precipitation of potassium perchlorate and subsequently filtered through a wet Whatman number 1 filter paper. Filters were washed with Milli-Q water and filtrates were eventually made up to a final volume of $25 \mathrm{~mL}$. Filtrates were stored at $-80^{\circ} \mathrm{C}$ until chromatographic analysis were performed.

The analysis of nucleotide catabolites was carried out by reverse-phase high performance liquid chromatography (RP-HPLC) as described in Vilas et al. (2017a).

Experimental data for nucleotide catabolites data are shown in Tables 1,3 , 5 and 7

Regarding bacterial analysis, we use the data presented in García et al. (2015). For the sake of completeness such data are included in A(Tables 2, 4, 6 and 8 . These data show that, at the end of the experiments, bacteria has already spoiled the fish. As mentioned in the introduction, the objective is to provide a model describing fish quality losses at early storage times (before bacteria spoilage is noticed). However, we extend the experiments time after spoilage because IMP, Ino and Hx are still present in the samples and they provide us with useful information for parameter estimation purposes. 


\section{The mathematical model}

The model we propose combines the most relevant enzymatic transformation routes of IMP, into Ino and Hx, as reported in Vilas et al. (2017a), with the catalytic effect of bacteria on some of those routes. Following Gram and Huss (1996); Gram and Dalgaard (2002), we assume that Pseudomonas and Shewanella are the predominant species involved in the spoilage of fresh fish. Their temperature dependent growth kinetics is given by García et al.(2015). In addition, the model incorporates the effect of the food matrix by considering diffusion of the resulting degradation products. As suggested in Howgate (2006), a first order leakage term is included to account for the effect of nucleotide's diffusion through fish muscle. Figure 2 provides a pictorial description of the different mechanisms in action during storage at low temperatures. The complete math-

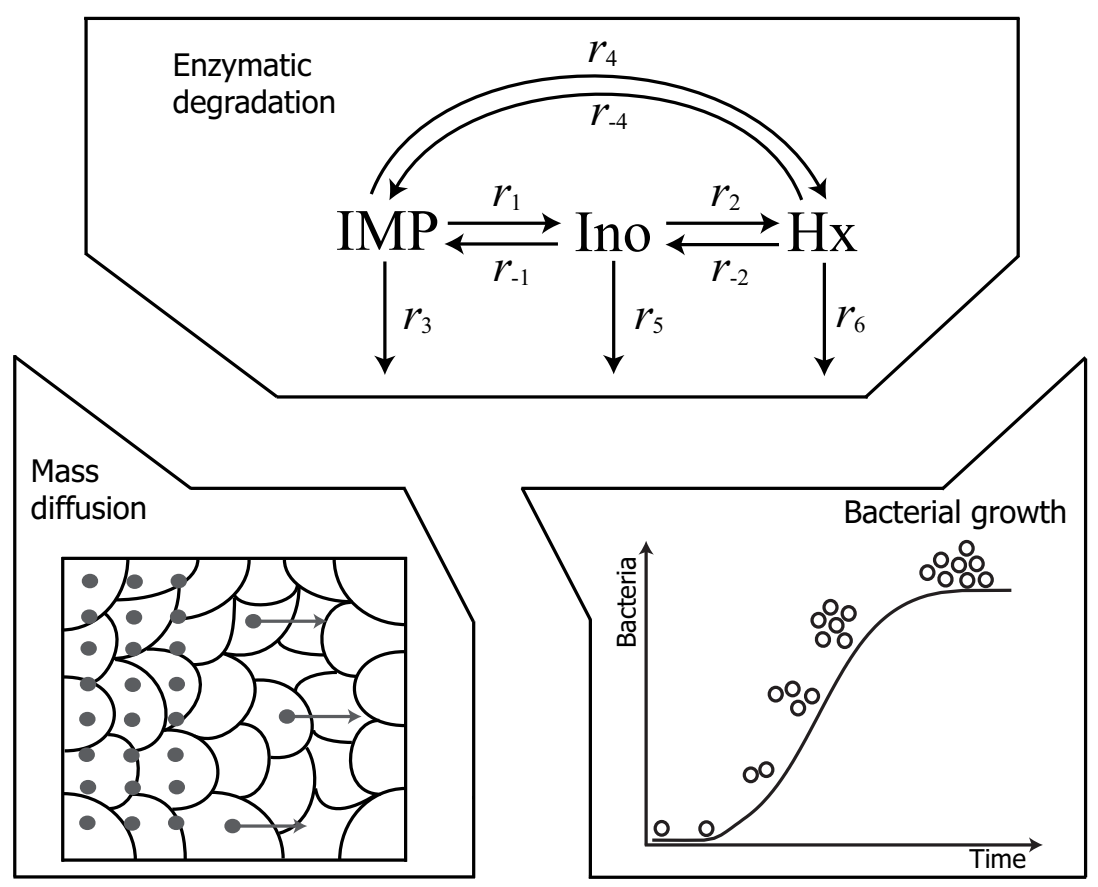

Figure 2: Combination, in a modular way, of the different mechanisms relevant in nucleotide degradation to obtain the complete model.

ematical model resulting from combining all the mechanisms is presented in $B$. 
The kinetics underlying the proposed mechanisms together with the methods employed for model calibration are given next.

\subsection{Biochemical degradation routes for IMP}

Figure 2 shows all possible enzymatic degradation routes mentioned in the literature. It should be noted that, as shown in Vilas et al. (2017a), many of such reactions are not relevant in European hake (Merluccius merluccius) at typical storage conditions. In this work, we consider a reduced version of the complete scheme of Figure 2 to describe the enzymatic degradation of IMP into Ino and HX. Such scheme was derived in Vilas et al. (2017a) and it is depicted in Figure 3.

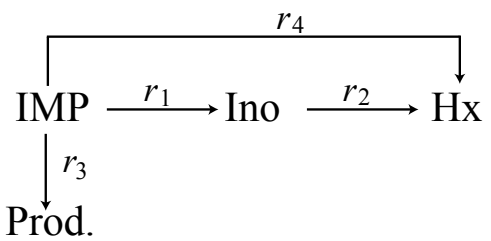

Figure 3: Postmortem degradation scheme of IMP in European hake as reported by Vilas et al. (2017a).

The model assumes that enzymatic degradation of IMP, Ino and Hx follows first order kinetics, so the time evolution of their corresponding concentrations can be described by the following set of ordinary differential equations:

$$
\begin{gathered}
\frac{\mathrm{d}[\mathrm{IMP}]}{\mathrm{d} t}=-\left(r_{1}+r_{3}+r_{4}\right) \\
\frac{\mathrm{d}[\mathrm{Ino}]}{\mathrm{d} t}=r_{1}-r_{2} \\
\frac{\mathrm{d}[\mathrm{Hx}]}{\mathrm{d} t}=r_{4}+r_{2}
\end{gathered}
$$

where $r_{1}=k_{1}[\mathrm{IMP}], r_{2}=k_{2}[\mathrm{Ino}], r_{3}=k_{3}[\mathrm{IMP}]$ and $r_{4}=k_{4}[\mathrm{IMP}]$. We assume that the reaction rate coefficients $\left(k_{i}\right)$ vary with the storage temperature according to an Arrhenius expression:

$$
k_{i}=A_{i} \exp \left(-\frac{E_{a, i}}{R T}\right)
$$


with $R=1.987 \mathrm{kcal} \mathrm{K}^{-1} \mathrm{kmol}^{-1}$ being the universal gas constant and $T$ in $\mathrm{K}$ the storage temperature, in general time variable. The values of the activation energies $\left(E_{a, i}\right)$ and pre-exponential factor $\left(A_{i}\right)$, as estimated in Vilas et al.(2017a), are presented in Table 1

\begin{tabular}{lll}
\hline Parameter & Value & Units \\
\hline$E_{a, 1}$ & $2.77 \times 10^{4}$ & $\mathrm{kcal} \mathrm{kmol}^{-1}$ \\
$A_{1}$ & $1.15 \times 10^{20}$ & $\mathrm{~d}^{-1}$ \\
$E_{a, 2}$ & $2.18 \times 10^{4}$ & $\mathrm{kcal} \mathrm{kmol}^{-1}$ \\
$A_{2}$ & $7.63 \times 10^{15}$ & $\mathrm{~d}^{-1}$ \\
$E_{a, 3}$ & $2.56 \times 10^{4}$ & $\mathrm{kcal} \mathrm{kmol}^{-1}$ \\
$A_{3}$ & $2.29 \times 10^{18}$ & $\mathrm{~d}^{-1}$ \\
$E_{a, 4}$ & $1.81 \times 10^{4}$ & $\mathrm{kcal} \mathrm{kmol}^{-1}$ \\
$A_{4}$ & $4.84 \times 10^{12}$ & $\mathrm{~d}^{-1}$ \\
\hline
\end{tabular}

Table 1: Values of the Arrhenius equation coefficients for reaction rates $r_{1}, r_{2}$ and $r_{4}$, as estimated in Vilas et al. (2017a).

\subsection{Effect of bacterial action}

As we mention in the introduction, and Figure 1 illustrates, the presence of bacteria in the system speeds up both the degradation of IMP and formation of Hx. This suggests a catalytic effect of bacteria on some of the biochemical routes given in Figure 3

In this work we study the potential catalytic effect of Pseudomonas and Shewanella bacterial populations on the reaction rates $r_{1}, r_{2}$ and $r_{4}$, that link [IMP], [Ino] and [Hx] in the pathway depicted in Figure 3. We assume that the rate of conversion under bacterial action is proportional to the number of microorganisms and the concentration of the corresponding compound to be degraded, so that:

$$
\begin{aligned}
& r_{1, b a c}=k_{1, b a c}(\mathrm{Sh}+\mathrm{Ps})[\mathrm{IMP}] \\
& r_{2, b a c}=k_{2, b a c}(\mathrm{Sh}+\mathrm{Ps})[\mathrm{Ino}] \\
& r_{4, b a c}=k_{4, b a c}(\mathrm{Sh}+\mathrm{Ps})[\mathrm{IMP}]
\end{aligned}
$$

Parameters to be estimated are the reaction rate coefficients $k_{i, b a c}$ with $i=1,2,4$. 
Bacteria catalytic effects $\left(r_{i, b a c}\right)$ will be added to the enzymes catalytic effects and $\left(r_{i}\right)$ when considering the complete model (see B for details).

Eqns (5)-(7) depend on the concentration of Pseudomonas and Shewanella, therefore, a model describing its time evolution is required. We adopt the model proposed in García et al. (2015) to describe bacterial growth. It makes use of the standard logistic model (Baranyi and Roberts, 1994):

$$
\begin{aligned}
\frac{\mathrm{dPs}}{\mathrm{d} t} & =\log (10) \mu_{P_{S}} \operatorname{Ps}\left(1-\frac{\mathrm{Ps}}{\mathrm{Ps}^{*}}\right) \\
\frac{\mathrm{dSh}}{\mathrm{d} t} & =\log (10) \mu_{S h} \mathrm{Sh}\left(1-\frac{\mathrm{Sh}}{\mathrm{Sh}^{*}}\right)
\end{aligned}
$$

where Ps and Sh refer to the concentration of Pseudomonas and Shewanella in $\mathrm{CFUg}^{-1}$. Growth rates $\left(\mu_{P_{s}}, \mu_{S h}\right)$ are described using the standard square-root model (Ratkowsky et al. 1982):

$$
\begin{aligned}
& \mu_{P_{S}}(T)=b_{P_{S}}^{2}\left(T-T_{p s}^{*}\right)^{2} \\
& \mu_{S h}(T)=b_{S h}^{2}\left(T-T_{S h}^{*}\right)^{2}
\end{aligned}
$$

where $b_{x}$ and $T_{x}^{*}$ with $x=$ Ps, Sh, are given parameters.

In this work we make use of the parameters obtained by García et al. (2015) for European hake under typical storage conditions. Their values are shown in Table2

\begin{tabular}{lll}
\hline Parameter & Value & Units \\
\hline$P_{S^{*}}$ & $3.16 \times 10^{6}$ & $\mathrm{CFUg}^{-1}$ \\
$b_{P S}^{2}$ & $9.06 \times 10^{-4}$ & $\mathrm{~d}^{-1}{ }^{\circ} \mathrm{C}^{-2}$ \\
$T_{P_{S}}^{*}$ & -26 & ${ }^{\circ} \mathrm{C}$ \\
$S^{*}$ & $1.82 \times 10^{6}$ & $\mathrm{CFUg}^{-1}$ \\
$b_{S h}^{2}$ & $2.10 \times 10^{-3}$ & $\mathrm{~d}^{-1}{ }^{\circ} \mathrm{C}^{-2}$ \\
$T_{S h}^{*}$ & -14 & ${ }^{\circ} \mathrm{C}$ \\
\hline
\end{tabular}

Table 2: Parameters of the square-root model (Eqns 10 - 97) for Pseudomonas and Shewanella in European hake under storage conditions, as reported by García et al. [2015). 


\subsection{Nucleotides leaching}

Following Howgate (2006), leaching of nucleotides through fish muscle is a two-phase process: diffusion through the muscle and skin and removal of solute in the leachate. However, diffusion is the limiting step. Besides, assuming that nucleotide concentration in the skin is zero, diffusion process can be approximated by first order reaction kinetics, so that:

$$
\frac{\mathrm{d}[\mathrm{Nuc}]}{\mathrm{d} t}=-D_{\mathrm{Nuc}}[\mathrm{Nuc}]
$$

where $[\mathrm{Nuc}]=\{[\mathrm{IMP}],[\mathrm{Ino}],[\mathrm{Hx}]\}$. In this work, we consider the same diffusion coefficient $D=D_{I M P}=D_{\text {Ino }}=D_{H x}$ since differences are not expected to be large (Howgate, 2006).

\subsection{Model calibration}

Model parameter values are obtained by minimizing the distance between model predictions and experimental data. We have chosen the maximum likelihood as the measure of such distance (Walter and Pronzato 1997). The advantage of this approach is that it allows taking into account the available information on the nature of the experimental noise. Finding the maximum likelihood is equivalent to minimize the following function:

$$
J_{l l k}(\theta)=-\frac{1}{2} \sum_{k=1}^{n_{c}} \sum_{i=1}^{n_{x_{k}}}\left[\log (2 \pi)+\log \left(\sigma_{x_{k, i}}^{2}\right)+\left(\frac{x_{k, i}(\theta)-x_{k, i}^{\exp }}{\sigma_{x_{k, i}}}\right)^{2}\right]
$$

where $n_{c}=3$ is the number of compounds (IMP, Ino, Hx), $x_{k}$ represents the concentration of each of the considered compounds (i.e. $x_{1}=[\mathrm{IMP}], x_{2}=[\mathrm{Ino}]$ and $\left.x_{3}=[\mathrm{Hx}]\right) . n_{x_{k}}$ the number of sampling points per component (for instance $n_{x_{1}}$ is the number of time measurements for [IMP]). Vector $\theta$ includes all unknown model parameters to be estimated and $x_{k, i}(\theta), x_{k, i}^{\exp }$ indicate the concentration of compound $x_{k}$ at time $t_{i}$ computed from the model, and measured in the experiments, respectively. $\sigma_{x_{k, i}}^{2}$ is the standard deviation of compound $x_{k}$ at time $t_{i}$. 
Parameters are estimated via a numerical solver, by finding the vector $\theta$ that minimizes $J_{l l k}(\theta)$ subject to the model dynamics (1)- (6). It is often the case that the objective function $J_{l l k}(\theta)$ has several minima (Vilas et al., 2017b) (i.e. suboptimal solutions) so, in order to efficiently reach the global minimum, we use a hybrid (global and local) optimization method (Egea et al. 2009). The toolbox AMIGO2 (Balsa-Canto et al. 2016) is used to find the minimum of $J_{l l k}(\theta)$.

Typically, when a set of models is available to describe a given process the best one is selected based on the best value of the objective function. In this way, one would keep the model that provides the lowest $J_{l k}$.

When nested models are at hand, as it is the case, the best fit corresponds to the model with the largest number of parameters. However, differences in the solution might be caused by the capacity of the more complex model to fit measurement noise. This phenomena is known as overfitting (Ljung, 1999; Gabor et al. 2017) and it should be avoided. Akaike's Information Criterion (AIC) can be used to compare nested models (Glatting et al. 2007) and prevent overfitting, as it penalizes those with large sets of parameters. When the number of data is small, as it is the case in this work, a correction term must be added to the AIC. For the objective function (13), the corrected AIC reads (Burnham et al. 2011):

$$
\mathrm{AIC}_{c}=J_{l l k}+\frac{2 n_{p}\left(n_{d}+2\right)}{n_{d}-n_{p}+1}
$$

where $n_{d}$ is number of measurements and $n_{p}$ is the number of parameters considered. One should select the model with the lowest $\mathrm{AIC}_{c}$ value.

\subsection{Practical identifiability analysis}

The solution of the parameter estimation problem should include a measure of the parameter reliability. Practical identifiability analysis is an important step in the parameter estimation problem intended to evaluate parameter uncertainty. Given a set of experimental data, uncertainty is evaluated in terms of confidence intervals. For a given parameter $\left(\theta_{i}\right)$, such interval may be obtained through 
the covariance matrix $(C)$ as follows:

$$
\pm t_{\alpha / 2}^{\gamma} \sqrt{C_{i i}}
$$

where $t_{\alpha / 2}^{\gamma}$ is given by Student's t-distribution, $\gamma$ corresponds to the number of degrees of freedom and $\alpha$ is the (1- $\alpha$ ) $100 \%$ confidence interval selected, typically $95 \%$ is used.

For non-linear systems, as the one considered in this work, the Cramér-Rao inequality is used as a bound of the covariance matrix (Walter and Pronzato, 1997: Ljung, 1999). In this way, and under some assumptions, matrix $C$ is bounded by the Fisher information matrix $(\mathcal{F})$ :

$$
\boldsymbol{C} \geq \mathcal{F}^{-1} ; \quad \text { with } \quad \mathcal{F}=E\left\{\left[\frac{\partial J_{l l k}(\boldsymbol{\theta})}{\partial \boldsymbol{\theta}}\right]\left[\frac{\partial J_{l l k}(\boldsymbol{\theta})}{\partial \boldsymbol{\theta}}\right]^{T}\right\}
$$

Correlation by pairs of parameters $\left(\theta_{i}, \theta_{j}\right)$ can be assessed as:

$$
C_{i j}^{r}=\frac{C_{i j}}{\sqrt{C_{i i} C_{j j}}}
$$

Parameters $\theta_{i}$ and $\theta_{j}$ are highly correlated if $C_{i j}^{r}$ is close to \pm 1 and uncorrelated if $C_{i j}^{r}=0$.

In order to compute parameter correlation and confidence intervals we use the AMIGO2 toolbox (Balsa-Canto et al. 2016).

\section{Results and discussion}

The underlying mechanisms involved in IMP degradation (enzymatic and bacterial catalysis as well as nucleotide diffusion) were described in Section 3 The complete model that combines the three mechanisms is presented in $B$ In this section, such model is confronted with four sets of experimental data. Each set consists of time concentration series for IMP, Ino and Hx obtained from hake fish stored at different temperatures in the range of 1 to $11^{\circ} \mathrm{C}$. Experimental data are depicted in Tables 1 , 8

The first three experimental series have been employed to study the potential influence of Pseudomonas and Shewanella on the reaction rates of the pathway 
depicted in Figure 3, that link IMP, Ino and Hx. The remaining series will be employed afterwards for model validation.

Modeling approaches to describe catalysis of bacteria on nucleotide degradation typically assume influence only on the conversion of Ino to Hx (Howgate, 2006), producing an increase in its reaction rate $\left(r_{2}\right.$ in Figure 3). This assumption is incorporated in this work into model 1 (see Table 3). For the sake of comparison we also consider that no reaction is affected by bacteria (model 0 ).

However, our experimental data also point out to an increase in the degradation rate of IMP, as shown by the differences between sterile and non-sterile conditions in Figure 1. Recent experimental works support this idea (Zotos, 2010: Li et al. 2017) so, in order to take into account the influence of bacteria on IMP degradation rate, the following alternatives are examined: (i) effect on reaction rates $r_{1}$ and $r_{2}$ (model 2); (ii) effect on reaction rates $r_{1}, r_{2}$ and $r_{4}$ (model $3)$.

For each of the four models considered (Models 0, 1, 2 and 3), we estimate the reaction rate parameters in expressions (5)-(7) with the methods described in Section 3.4 These models can be regarded as nested models, what supports the use of the AIC criterion for comparison purposes. Table 3 shows the $A I C_{c}$, together with the number of parameters and the value of $J_{l l k}$, for each of the considered scenarios. The number of measurements in the estimation experiments is $n_{d}=63$. According to the $A I C_{c}$ criterion the best model, understood

\begin{tabular}{llccc}
\hline Model & $\begin{array}{l}\text { Reactions affected by } \\
\text { bacteria }\end{array}$ & $n_{p}$ & $J_{l l k}$ & $A I C_{c}$ \\
\hline 0 & - & 4 & 31.8 & 40.4 \\
1 & $r_{2}$ & 5 & 23.4 & 34.4 \\
2 & $r_{1}, r_{2}$ & 6 & -11 & 2.4 \\
3 & $r_{1}, r_{2}, r_{4}$ & 7 & -12.8 & 3.1 \\
\hline
\end{tabular}

Table 3: Values of the AIC for the different alternatives, involving the estimation of different reaction rate coefficients.

as that which better explains the experimental data without over-fitting, is the one that considers bacterial action only on $r_{1}$ and $r_{2}$, i.e. model 2 . In addition, 
two conclusions can be drawn: on the one hand, that bacterial action on $r_{4}$ can be neglected, since the small improvement in $J_{l l k}$ is obtained at the expenses of over-fitting. On the other hand, the improvement of model 2 over models 0 and 1 , in terms of the AIC and $J_{l l k}$, is significant (one order of magnitude).

In order to check possible correlation problems among parameters, we applied the procedure described in Section 3.5 to model 2. Correlation results by pair of parameters are:

$$
C_{k_{1, b a c}, k_{2, b a c}}^{r}=-0.036 ; \quad C_{k_{1, b a c}, D}^{r}=0.51 ; \quad C_{k_{2, b a c}, D}^{r}=0.32 ;
$$

All these coefficients are far from \pm 1 so correlation is low. This is confirmed when we draw the contour plots for each pair of parameters (Figure 4). Such contour plots represent the variation of $J_{l k k}$ as a function of two parameters. As shown in the figure, contours are rather round (low eccentricity) indicating low correlation.
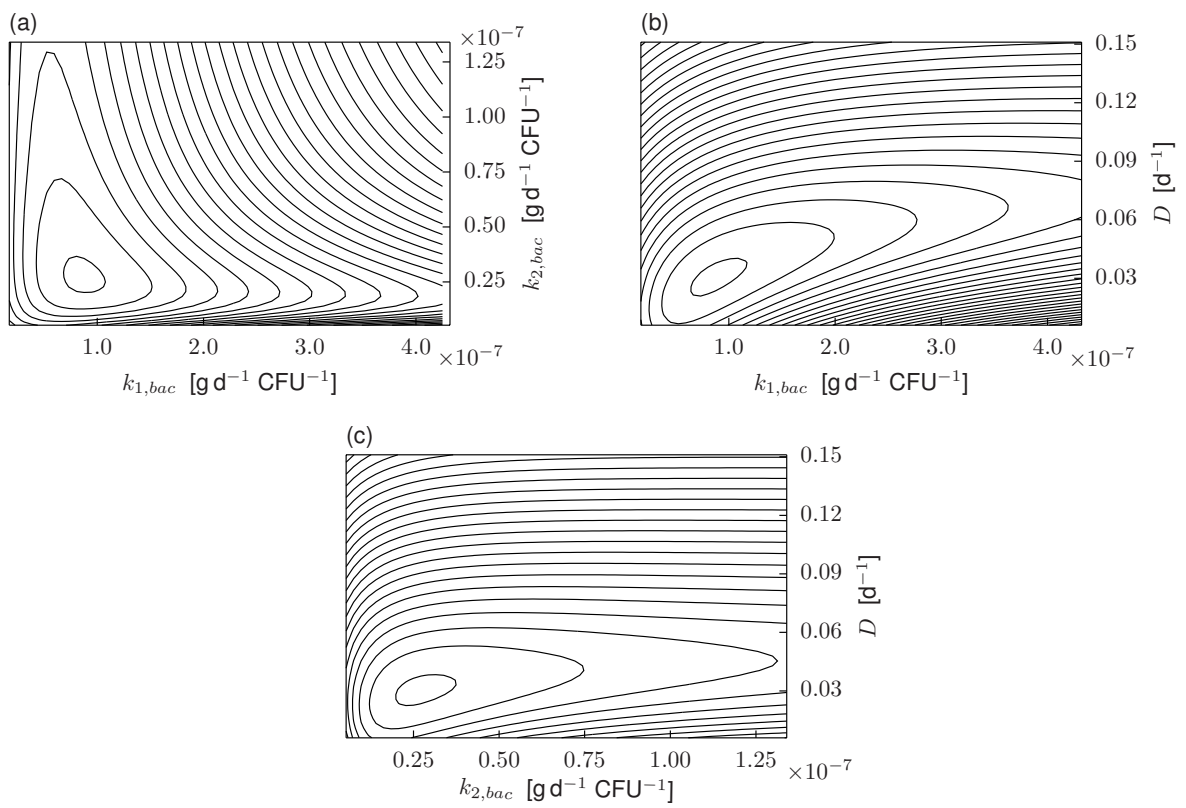

Figure 4: Contour plots of the objective function $\left(J_{l l k}\right)$ by pair of parameters.

Table 4 presents the estimated parameter values and their confidence intervals. Such intervals are considered satisfactory taking into account that they 
reflect all sources of variability, including fish-to-fish variability and measurement error (García et al., 2017).

\begin{tabular}{lll}
\hline Parameter & Value & Units \\
\hline$k_{1, b a c}$ & $(8.64 \pm 1.69) \times 10^{-8}$ & $\mathrm{~g} \mathrm{~d}^{-1} \mathrm{CFU}^{-1}$ \\
$k_{2, b a c}$ & $(2.68 \pm 0.61) \times 10^{-7}$ & $\mathrm{gd}^{-1} \mathrm{CFU}^{-1}$ \\
$D$ & $(3.02 \pm 0.74) \times 10^{-2}$ & $\mathrm{~d}^{-1}$ \\
\hline
\end{tabular}

Table 4: Estimated values of the global parameters for model 2. Initial conditions estimated per experiment are: $1.25 \mathrm{~mm}$ for experiment 1; $1.47 \mathrm{~mm}$ for experiment 2; and $1.01 \mathrm{~mm}$ for experiment 3

Finally, we make use of the experiment not considered in the parameter estimation problem to validate the model and illustrate its predictive capabilities. The comparison of model solution vs experimental data is presented in Figure 5 As shown in the figure, experimental data indicate an increase in

(a)
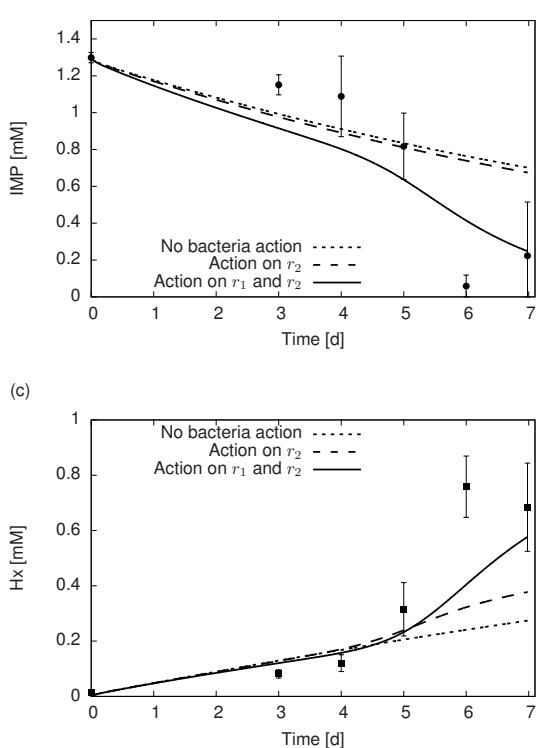

(b)

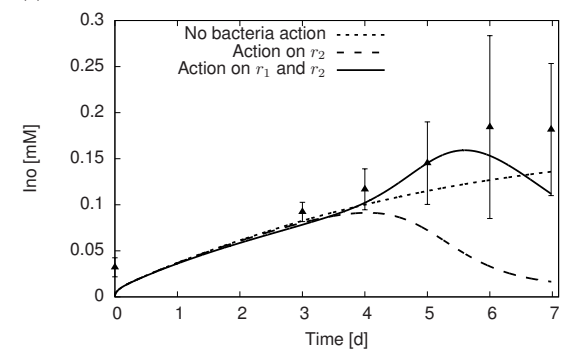

(d)

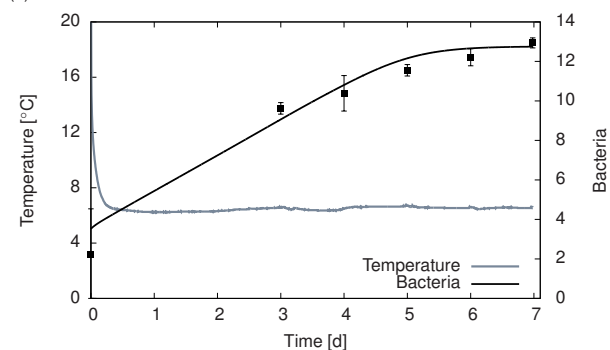

Figure 5: (a)-(c) Comparison between the solution of models 0, 1 and 2 and experimental data for the validation experiment. Marks represent experimental data. (d) Storage temperature (gray continuous line) and bacterial evolution (Ps+Sh) in logarithmic scale as predicted by the growth model (black continuous line) and experimentally measured (marks).

the formation rate of $\mathrm{Hx}$ and in the degradation rate of IMP around days 4 
and 5 (when bacteria concentration is large). The model selected in this work (model 2, continuous lines) is able to capture this behavior. However, when bacteria catalysis is restricted to $r_{2}$ (model 1 , dashed lines), the model is not able to capture the IMP degradation rate increase and wrongly predicts a rapid consumption of Ino. Considering bacterial effect on $r_{1}$ compensates Ino consumption to form $\mathrm{Hx}$ with formation of Ino from IMP, as the experimental data suggest.

\section{Conclusions}

In this work we used a modular approach to develop a model that describes the evolution of nucleotides typically used as early quality indicators (IMP, Ino and Hx) during storage and transport conditions. The model is composed of three elements: nucleotide enzymatic degradation; catalytic effect of bacteria and diffusion through the fish muscle. Although the model was developed and validated for European Hake, modularity provides us with the flexibility to adapt it to other species and storage conditions.

We found experimental evidences that bacteria have a large effect on both Hx formation and IMP degradation. Different modeling alternatives were used to explain such effect. These alternatives were compared in terms of the Akaike Information Criterion to avoid overfitting. The comparison led to the conclusion that bacteria mainly catalyze two steps of the degradation pathway, namely, the transformation from IMP to Ino and from Ino to Hx. This conclusion differs from usual modeling approaches considering that bacteria only catalyzes the transformation from Ino to Hx. Our results also showed negligible bacterial catalysis on the direct transformation of IMP to Hx.

Unknown model parameters were estimated from three sets of experimental data. A fourth set, carried out at different storage conditions, was used for model validation. The proposed model was able to satisfactorily reproduce the validation data. 


\section{Acknowledgements}

The authors acknowledge financial support from the Spanish Ministry of Science and Innovation (Projects ISFORQUALITY AGL2012-39951-C02-01 and RESISTANCE DPI2014-54085-JIN).

\section{References}

Balsa-Canto, E., Henriques, D., Gabor, A., and Banga, J. R. (2016). AMIGO2, a toolbox for dynamic modeling, optimization and control in systems biology. Bioinformatics. DOI:10.1093/bioinformatics/btw411.

Baranyi, J. and Roberts, T. A. (1994). A dynamic approach to predicting bacterial growth in food. International Journal of Food Microbiology, 23:277-294.

Beveridge, T. J. and Kadurugamuwa, J. L. (1996). Periplasm, periplasmic spaces, and their relation to bacterial wall structure: novel secretion of selected periplasmic proteins from pseudomonas aeruginosa. Microb Drug Resist, 2(1):1-8.

Burnham, K. P., Anderson, D. R., and Huyvaert, K. P. (2011). AIC model selection and multimodel inference in behavioral ecology: some background, observations, and comparisons. Behavioral Ecology and Sociobiology, 65(1):2332.

Davídek, J., Velíšek, J., and Janíček, G. (1972). Stability of inosinic acid, inosine and hypoxanthine in aqueous solutions. Journal of Food Science, 37(5):789-790.

Egea, J. A., Vazquez, E., Banga, J. R., and Marti, R. (2009). Improved scatter search for the global optimization of computationally expensive dynamic models. Journal of Global Optimization, 43(2-3):175-190.

Gabor, A., Villaverde, A. F., and Banga, J. R. (2017). Parameter identifiability analysis and visualization in large-scale kinetic models of biosystems. BMC systems biology, 11:1-16. 
García, M. R., Cabo, M. L., Herrera, J. R., Ramilo-Fernández, G., Alonso, A. A., and Balsa-Canto, E. (2017). Smart sensor to predict retail fresh fish quality under ice storage. Journal of Food Engineering, 197:87-97.

García, M. R., Vilas, C., Herrera, J. R., Bernárdez, M., Balsa-Canto, E., and Alonso, A. A. (2015). Quality and shelf-life prediction for retail fresh hake (merluccius merluccius). International Journal of Food Microbiology, 208:65-74.

Giuffrida, A., Valenti, D., Ziino, G., , and Panebianco, A. (2009a). Study on the application of an interspecific competition model for the prediction of microflora behaviour during the fermentation process of s. angelo PGI salami. Veterinary Research Communications, 33:S229-S232.

Giuffrida, A., Valenti, D., Ziino, G., Spagnolo, B., and Panebianco, A. (2009b). A stochastic interspecific competition model to predict the behaviour of Listeria monocytogenes in the fermentation process of a traditional sicilian salami. European Food Research and Technology, 228(5):767-775.

Giuffrida, A., Ziino, G., Valenti, D., Donato, G., and Panebianco, A. (2007). Application of an interspecific competition model to predict the growth of Aeromonas hydrophila on fish surfaces during refrigerated storage. Archiv fur Lebensmittelhygiene, 58(4):136-141.

Glatting, G., Kletting, P., Reske, S. N., Hohl, K., and Ring, C. (2007). Choosing the optimal fit function: Comparison of the Akaike information criterion and the F-test. Medical Physics, 24(11):4285-4292.

Gram, L. (1993). Inhibitory effect against pathogenic and spoilage bacteria of pseudomonas strains isolated from spoiled and fresh fish. Applied and Environmental Microbiology, 59(7):2197-2203.

Gram, L. and Dalgaard, P. (2002). Fish spoilage bacteria-problems and solutions. Current Opinion in Biotechnology, 13(3):262-266.

Gram, L. and Huss, H. H. (1996). Microbiological spoilage of fish and fish products. International Journal of Food Microbiology, 33(1):121-137. 
Gram, L. and Melchiorsen, J. (1996). Interaction between fish spoilage bacteria pseudomonas sp. and shewanella putrefaciens in fish extracts and on fish tissue. Journal of Applied Bacteriology, 80(6):589-595.

Haard, N. (2002). The role of enzymes in determining seafood color, flavour and texture. In Bremmer, H. A., editor, Safety and Quality Issues in Fish Processing, pages 220-253. Woodhead Publishing Limited.

Hara, A. and Uda, F. (1984). Theoretical and experimental studies on the timetemperature tolerance of fish muscle K value. Bulletin of the Japanese Society of Scientific Fisheries, 50:1745-1756.

Hong, H., Regenstein, J. M., and Luo, Y. (2017). The importance of ATP-related compounds for the freshness and flavor of post-mortem fish and shellfish muscle: A review. Critical Reviews in Food Science and Nutrition, 59(9):17871798.

Howgate, P. (2006). A review of the kinetics of degradation of inosine monophosphate in some species of fish during chilled storage. International Journal of Food Science and Technology, 41:341-353.

Ishida, Y., Tsuruta, H., Tsuneta, S. T., Uno, T., Watanabe, K., and Aizono, Y. (1998). Characteristics of psychrophilic alkaline phosphatase. Bioscience, Biotechnology and Biochemistry, 62(11):2246-2250.

Karube, I., Matsuoka, H., Suzuki, S., Watanabe, E., and Toyama, K. (1984). Determination of fish freshness with an enzyme sensor system. Journal of agricultural and food chemistry, 32(2):314-319.

Kawai, M., Okiyama, A., and Ueda, Y. (2002). Taste enhancements between various amino acids and imp. Chemical Senses, 27:739-745.

Kuda, T., Fujita, M., Goto, H., and Yano, T. (2008). Effects of retort conditions on ATP-related compounds in pouched fish muscle. Food Science and Technology, 41(3):469-473. 
Kuribayashi, T. A., Fujii, S., Masanari, M., Yamanaka, M., Wakai, S., and Sambongi, Y. (2017). Difference in $\mathrm{NaCl}$ tolerance of membrane-bound 5'nucleotidases purified from deep-sea and brackish water shewanella species. Extremophiles, 21(2):257-268.

Lazdunski A, Guzzo J, F. A. B. M. M. M. (1990). Secretion of extracellular proteins by pseudomonas aeruginosa. Biochimie, 72(2-3):147-156.

Li, D., Zhang, L., Song, S., Wang, Z., Kong, C., and Luo, Y. (2017). The role of microorganisms in the degradation of adenosine triphosphate (ATP) in chillstored common carp (cyprinus carpio) fillets. Food Chemistry, 224(1):347-352.

Li, K. F., Luo, Y. K., and Shen, H. X. (2015). Postmortem changes of crucian carp (carassius auratus) during storage in ice. International Journal of Food Properties, 18(1):205-212.

Ljung, L. (1999). System Identification: Theory for the User. Prentice Hall PTR.

Özogul, F., Taylor, K. D. A., Quantick, P. C., and Özogul, Y. (2000). A rapid hplc-determination of atp-related compounds and its application to herring stored under modified atmosphere. International Journal of Food Science and Technology, 35(6):549-554.

Ratkowsky, D., Olley, J., McMeekin, T., and Ball, A. (1982). Relationship between temperature and growth rate of bacterial cultures. Journal of Bacteriology, 149(1):1-5.

Saito, T., Arai, K., and Matsuyoshi, M. (1959). A new method for estimating the freshness of fish. Bulletin of the Japanese Society of Scientific Fisheries, 24:749-750.

Surette, M. E., Gill, T. A., and LeBlanc, P. J. (1988). Biochemical basis of postmortem nucleotide catabolism in cod (gadus morhua) and its relationship to spoilage. Journal of agricultural and food chemistry, 36(1):19-22.

Tsuruta, H., Mikami, B., Higashi, T., and Aizono, Y. (2010). Crystal structure of cold-active alkaline phosphatase from the psychrophile shewanella sp. Bioscience, Biotechnology and Biochemistry, 74(1):29-74. 
Vilas, C., Alonso, A. A., Herrera, J. R., García-Blanco, A., and García, M. R. (2017a). A model for the biochemical degradation of inosine monophosphate in hake (Merluccius merluccius). Journal of Food Engineering, 200:95-101.

Vilas, C., Arias-Méndez, A., García, M. R., Alonso, A. A., and BalsaCanto, E. (2017b). Towards predictive food process models: A protocol for parameter estimation. Critical Reviews in Food Science and Nutrition. DOI:10.1080/10408398.2016.1186591.

Walter, E. and Pronzato, L. (1997). Identification of parametric models from experimental data. Springer.

Zimmermann, H. (1992). 5'-nucleotidase: molecular structure and functional aspects. Biochem. J., 285:345-365.

Zotos, A. (2010). Preservation time at $6 \pm 2 \mathrm{C}$ and $8 \pm 2 \mathrm{C}$ of trout (Salmo gairdnerii) fillets smoked by steaming with liquid smoke. Journal of Food Processing and Preservation, 35:533-541.

\section{A Experimental data}

In this appendix, we include the tables containing the experimental data employed to calibrate and validate models presented in this work. See Tables 1.8.

\begin{tabular}{llll}
\hline Time [days] & IMP $[\mathrm{mm}]$ & Ino $[\mathrm{mm}]$ & Hx $[\mathrm{mm}]$ \\
\hline 0.0 & $1.299 \pm 0.028$ & $0.032 \pm 0.010$ & $0.014 \pm 0.004$ \\
3.0 & $1.252 \pm 0.024$ & $0.066 \pm 0.001$ & $0.048 \pm 0.024$ \\
5.0 & $1.173 \pm 0.033$ & $0.107 \pm 0.011$ & $0.081 \pm 0.004$ \\
7.0 & $0.891 \pm 0.164$ & $0.096 \pm 0.002$ & $0.084 \pm 0.012$ \\
10.0 & $0.664 \pm 0.043$ & $0.146 \pm 0.027$ & $0.308 \pm 0.022$ \\
12.0 & $0.710 \pm 0.190$ & $0.131 \pm 0.007$ & $0.318 \pm 0.021$ \\
\hline
\end{tabular}

Table 1: Measured values and standard deviation of nucleotides for the first experiment, carried out at $1{ }^{\circ} \mathrm{C}$. This experiment was used for estimation purposes. 


\begin{tabular}{lll}
\hline Time [days] & Ps $\left[\log \left(\mathrm{CFUg}^{-1}\right)\right]$ & $\mathrm{Sh}\left[\log \left(\mathrm{CFUg}^{-1}\right)\right]$ \\
\hline 0.0 & $1.246 \pm 1.1957$ & $1.429 \pm 1.0404$ \\
3.0 & $2.089 \pm 1.4570$ & $3.406 \pm 0.1431$ \\
5.0 & $3.957 \pm 0.2627$ & $4.504 \pm 0.1890$ \\
7.0 & $4.818 \pm 0.2371$ & $5.482 \pm 0.1445$ \\
10.0 & $6.151 \pm 0.3729$ & $6.389 \pm 0.2434$ \\
12.0 & $6.277 \pm 0.3025$ & $6.671 \pm 0.0806$ \\
\hline
\end{tabular}

Table 2: Measured values and standard deviation of bacteria for the first experiment, carried out at $1{ }^{\circ} \mathrm{C}$. This experiment was used for estimation purposes.

\begin{tabular}{llll}
\hline Time [days] & IMP $[\mathrm{mm}]$ & Ino $[\mathrm{mm}]$ & Hx $[\mathrm{mm}]$ \\
\hline 0.0 & $1.313 \pm 0.052$ & $0.038 \pm 0.038$ & $0.026 \pm 0.010$ \\
1.0 & $1.079 \pm 0.330$ & $0.051 \pm 0.006$ & $0.141 \pm 0.149$ \\
2.0 & $1.328 \pm 0.006$ & $0.073 \pm 0.012$ & $0.033 \pm 0.008$ \\
5.0 & $0.742 \pm 0.200$ & $0.264 \pm 0.149$ & $0.176 \pm 0.033$ \\
6.0 & $0.798 \pm 0.145$ & $0.231 \pm 0.134$ & $0.154 \pm 0.036$ \\
7.0 & $0.336 \pm 0.320$ & $0.189 \pm 0.074$ & $0.507 \pm 0.285$ \\
8.0 & $0.158 \pm 0.134$ & $0.162 \pm 0.101$ & $0.621 \pm 0.019$ \\
\hline
\end{tabular}

Table 3: Measured values of nucleotides for the second experiment, carried out at $2.5^{\circ} \mathrm{C}$. This experiment was used for estimation purposes.

\begin{tabular}{lll}
\hline Time [days $]$ & Ps $\left[\log \left(\mathrm{CFUg}^{-1}\right)\right]$ & $\mathrm{Sh}\left[\log \left(\mathrm{CFUg}^{-1}\right)\right]$ \\
\hline 0.0 & $0.792 \pm 0.9435$ & $1.015 \pm 0.9694$ \\
1.0 & $0.928 \pm 1.0784$ & $2.417 \pm 0.1731$ \\
2.0 & $1.900 \pm 0.2329$ & $3.030 \pm 0.1626$ \\
5.0 & $5.234 \pm 0.0650$ & $5.293 \pm 0.1995$ \\
6.0 & $6.237 \pm 0.1374$ & $6.066 \pm 0.1532$ \\
7.0 & $6.055 \pm 0.1655$ & $5.900 \pm 0.1621$ \\
8.0 & $6.400 \pm 0.2390$ & $5.782 \pm 0.2045$ \\
\hline
\end{tabular}

Table 4: Measured values of bacteria for the second experiment, carried out at $2.5{ }^{\circ} \mathrm{C}$. This experiment was used for estimation purposes. 


\begin{tabular}{llll}
\hline Time [days] & IMP $[\mathrm{mm}]$ & Ino $[\mathrm{mm}]$ & $\mathbf{H x}[\mathrm{mm}]$ \\
\hline 0.0 & $0.906 \pm 0.113$ & $0.016 \pm 0.020$ & $0.005 \pm 0.020$ \\
1.0 & $0.845 \pm 0.077$ & $0.033 \pm 0.009$ & $0.000 \pm 0.009$ \\
2.0 & $0.719 \pm 0.011$ & $0.035 \pm 0.043$ & $0.019 \pm 0.043$ \\
5.0 & $0.697 \pm 0.072$ & $0.084 \pm 0.005$ & $0.031 \pm 0.005$ \\
6.0 & $0.778 \pm 0.094$ & $0.085 \pm 0.014$ & $0.011 \pm 0.014$ \\
7.0 & $0.750 \pm 0.079$ & $0.108 \pm 0.030$ & $0.093 \pm 0.030$ \\
8.0 & $0.572 \pm 0.140$ & $0.118 \pm 0.026$ & $0.146 \pm 0.026$ \\
9.0 & $0.067 \pm 0.106$ & $0.114 \pm 0.070$ & $0.512 \pm 0.070$ \\
\hline
\end{tabular}

Table 5: Measured values of nucleotides for the third experiment, carried out at 0.5 and $11^{\circ} \mathrm{C}$. This experiment was used for estimation purposes.

\begin{tabular}{lll}
\hline Time [days $]$ & Ps $\left[\log \left(\mathrm{CFUg}^{-1}\right)\right]$ & $\mathrm{Sh}\left[\log \left(\mathrm{CFUg}^{-1}\right)\right]$ \\
\hline 0.0 & $1.646 \pm 0.963$ & $2.479 \pm 0.197$ \\
1.0 & $0.808 \pm 0.977$ & $2.308 \pm 0.302$ \\
2.0 & $1.120 \pm 1.002$ & $2.578 \pm 0.283$ \\
5.0 & $4.098 \pm 0.501$ & $4.708 \pm 0.369$ \\
6.0 & $4.797 \pm 0.281$ & $5.090 \pm 0.110$ \\
7.0 & $5.818 \pm 0.173$ & $5.765 \pm 0.450$ \\
8.0 & $6.467 \pm 0.170$ & $6.238 \pm 0.231$ \\
9.0 & $6.214 \pm 0.329$ & $5.787 \pm 0.340$ \\
\hline
\end{tabular}

Table 6: Measured values of bacteria for the third experiment, carried out at 0.5 and $11{ }^{\circ} \mathrm{C}$. This experiment was used for estimation purposes.

\begin{tabular}{llll}
\hline Time [days] & IMP $[\mathrm{mm}]$ & Ino $[\mathrm{mM}]$ & Hx $[\mathrm{mm}]$ \\
\hline 0.0 & $1.299 \pm 0.028$ & $0.032 \pm 0.010$ & $0.014 \pm 0.004$ \\
3.0 & $1.151 \pm 0.054$ & $0.092 \pm 0.010$ & $0.081 \pm 0.015$ \\
4.0 & $1.088 \pm 0.219$ & $0.117 \pm 0.022$ & $0.120 \pm 0.030$ \\
5.0 & $0.817 \pm 0.180$ & $0.145 \pm 0.045$ & $0.315 \pm 0.097$ \\
6.0 & $0.059 \pm 0.059$ & $0.184 \pm 0.099$ & $0.759 \pm 0.111$ \\
7.0 & $0.223 \pm 0.292$ & $0.182 \pm 0.072$ & $0.684 \pm 0.159$ \\
\hline
\end{tabular}

Table 7: Measured values of nucleotides for the third experiment, carried out at $6.5{ }^{\circ} \mathrm{C}$. This experiment was used for validation purposes. 


\begin{tabular}{lll}
\hline Time [days] & Ps $\left[\log \left(\mathrm{CFUg}^{-1}\right)\right]$ & $\mathrm{Sh}\left[\log \left(\mathrm{CFUg}^{-1}\right)\right]$ \\
\hline 0.0 & $1.246 \pm 1.196$ & $1.000 \pm 1.094$ \\
3.0 & $4.768 \pm 0.164$ & $4.855 \pm 0.128$ \\
4.0 & $5.216 \pm 0.322$ & $5.171 \pm 0.576$ \\
5.0 & $5.987 \pm 0.174$ & $5.564 \pm 0.117$ \\
6.0 & $6.247 \pm 0.195$ & $5.966 \pm 0.238$ \\
7.0 & $6.536 \pm 0.046$ & $6.403 \pm 0.209$ \\
\hline
\end{tabular}

Table 8: Measured values of bacteria for the third experiment, carried out at $6.5^{\circ} \mathrm{C}$. This experiment was used for validation purposes. 


\section{B The complete degradation model}

In this section we present the complete model for nucleotide degradation after including all the elements: enzymatic degradation; bacteria catalysis and diffusion through the food matrix. The equations describing IMP, Ino and Hx evolution are:

$$
\begin{gathered}
\frac{\mathrm{d}[\mathrm{IMP}]}{\mathrm{d} t}=-\left(r_{1}+r_{3}+r_{4}+r_{1, b a c}\right)-D[\mathrm{IMP}] \\
\frac{\mathrm{d}[\mathrm{Ino}]}{\mathrm{d} t}=r_{1}+r_{1, b a c}-\left(r_{2}+r_{2, b a c}\right)+D[\text { Ino }] \\
\frac{\mathrm{d}[\mathrm{Hx}]}{\mathrm{d} t}=r_{4}+r_{2}+r_{2, b a c}-D[\mathrm{Hx}]
\end{gathered}
$$

where

$$
\begin{gathered}
r_{1}=k_{1}[\mathrm{IMP}] ; \quad r_{2}=k_{2}[\mathrm{Ino}] ; \quad r_{3}=k_{3}[\mathrm{IMP}] ; \quad r_{4}=k_{4}[\mathrm{IMP}] \\
r_{1, b a c}=k_{1, b a c}(\mathrm{Ps}+\mathrm{Sh})[\mathrm{IMP}] ; \quad r_{2, b a c}=k_{2, b a c}(\mathrm{Ps}+\mathrm{Sh})[\mathrm{Ino}]
\end{gathered}
$$

Coefficients $k_{1}, k_{2}, k_{3}$ and $k_{4}$ are temperature dependent through the Arrhenius equation:

$$
k_{i}=A_{i} \exp \left(-\frac{E_{a, i}}{R T}\right) ; \quad \text { for } i=1,2,3,4
$$

Note that, in order to solve Eqns (18)-(20), a model for bacterial growth (Ps and $\mathrm{Sh}$ ) is required. Such model is of the form:

$$
\begin{aligned}
& \frac{\mathrm{dPs}}{\mathrm{d} t}=\log (10) \mu_{P_{S}} \mathrm{Ps}\left(1-\frac{\mathrm{Ps}}{\mathrm{Ps}^{*}}\right) \\
& \frac{\mathrm{dSh}}{\mathrm{d} t}=\log (10) \mu_{S h} \mathrm{Sh}\left(1-\frac{\mathrm{Sh}}{\mathrm{Sh}^{*}}\right)
\end{aligned}
$$

